\title{
Angular Distribution of GRBs
}

\author{
L. G. Balázs, A. Mészáros, I. Horváth, Z. Bagoly, P. Veres, G. Tusnády
}

\begin{abstract}
We studied the complete randomness of the angular distribution of BATSE gamma-ray bursts (GRBs). Based on their durations and peak fluxes, we divided the BATSE sample into 5 subsamples (short1, short2, intermediate, long1, long2) and studied the angular distributions separately. We used three methods to search for non-randomness in the subsamples: Voronoi tesselation, minimal spanning tree, and multifractal spectra. To study any non-randomness in the subsamples we defined 13 test-variables (9 from Voronoi tesselation, 3 from the minimal spanning tree and one from the multifractal spectrum). We made Monte Carlo simulations taking into account the BATSE's sky-exposure function. We tested the randomness by introducing squared Euclidean distances in the parameter space of the test-variables. We recognized that the short1, short2 groups deviate significantly $(99.90 \%, 99.98 \%)$ from the fully random case in the distribution of the squared Euclidean distances but this is not true for the long samples. In the intermediate group, the squared Euclidean distances also give significant deviation (98.51\%).
\end{abstract}

\section{Introduction}

Recently, the cosmological origin of gamma-ray bursts (hereafter GRBs) has been widely accepted $[24,49,75]$. Assuming large scale isotropy for the Universe, one would also expect the same property for GRBs.

There is an increasing amount of evidence that all GRBs do not all form a physically homogeneous group [5, 27-31, 39,50]. Hence, it is worth investigating whether physically different subgroups are also different in their angular distributions. The authors have carried out several different tests in recent years $[3,4,45,46]$ probing the intrinsic isotropy in the angular sky-distribution of the GRBs collected in BATSE Catalog [44]. Briefly summarizing the results of these studies, one may conclude: A. The long subgroup $\left(T_{90}>10 s\right)$ seems to be distributed isotropically; B. The intermediate subgroup $\left(2 s \leq T_{90} \leq 10 s\right)$ is distributed anisotropically on the $\simeq(96-97) \%$ significance level; C. For the short subgroup $\left(T_{90}<2 s\right)$ the assumption of isotropy is rejected only on the $92 \%$ significance level; D. The long and short subclasses, respectively, are distributed differently on the $99.3 \%$ significance level. (For a definition of the subclasses see [29-33,69].)

Independently and by different tests, [43] confirmed results A., B. and C., with one essential difference: for the intermediate subclass a much higher namely $99.89 \%$ - significance level of anisotropy is claimed. Again, the short subgroup is found to be "suspicious", but only the $\simeq(85-95) \%$ significance level is reached. The long subclass seems to be distributed isotropically. [42] found a significant angular correlation on the $2^{\circ}-5^{\circ}$ scale for GRBs of $T_{90}<2 s$ duration. [64] reported a correlation between the lo- cations of previously observed short bursts and the positions of galaxies in the local Universe, indicating that between 10 and 25 per cent of short GRBs originate at low redshifts $(z<0.025)$.

It is a reasonable requirement to continue these tests using more sophisticated procedures in order to see whether the angular distribution of GRBs is completely random, or whether it has some sort of regularity. This is the subject of this paper. New tests will be presented here. Mainly the clarification of the short subgroup's behaviour is expected from these tests. In this paper, similarly to the previous studies, the intrinsic randomness is tested; this means that the non-uniform sky-exposure function of BATSE instrument is eliminated.

The paper is organized as follows. The three new tests are described in Section 2. This Section does not contain new results, but this minimal survey may be useful because the methods are not widely familiar. Section 3 contains the statistical tests on the data. Section 4 summarizes the results of the statistical tests, and Section 5 presents the main conclusions of the paper. The paper is based on the results published in [68]; some preliminary cosmological implications are also discussed by $[47,48]$.

\section{Mathematical overview}

Strictly from the mathematical point of view, considering GRBs as a point process on the celestial sphere, the first property means that for a given $\Omega$ area on the sky the $P(\Omega)$ probability of observing a burst depends only on the size of $\Omega$, and not on its location on the sphere. The second property means that for the given two disjunct $\Omega_{1}, \Omega_{2}$ areas on the sky the joint probability is given by $P\left(\Omega_{1}, \Omega_{2}\right)=P\left(\Omega_{1}\right) P\left(\Omega_{2}\right)$, i.e. 
the probability of observing a burst in $\Omega_{1}$ is fully independent from the probability of getting one in $\Omega_{2}$.

If both properties are fulfilled, the distribution is called completely random (for the astronomical context of spatial point processes, see [55]). There are several tests for checking the complete randomness of point patterns, but these procedures do not always give information for both properties simultaneously.

The simplest test for isotropy is a comparison of the number counts of GRBs in disjunct areas on the sky. In the case of isotropy expanding $P(\Omega)$ into a series of spherical harmonics, all the coefficients in the series, except the 0th order, should equal zero. This property can also be used for testing the isotropy (for testing the dipole and quadrupole moments, see [10]). Before performing the isotropy tests the uneven exposure function of BATSE has to be taken into account [44].

We used three methods, Voronoi tesselation, minimal spanning tree and multifractal spectra to get statistical variables suitable for testing the fully randomness of the angular distribution of GRBs.

\subsection{Voronoi tesselation (VT)}

The Voronoi diagram - also known as Dirichlet tesselation or Thiessen polygons - is a fundamental structure in computational geometry and arises naturally in many different applications [70,62]. Generally, this diagram provides a partition of a point pattern ("point field", also "point process"), according to its spatial structure, which can be used for analyzing the underlying point process.

Let us assume that there are $N$ points $(N \gg 1)$ scattered on a sphere surface with a unit radius. We say that a point field is given on the sphere. The Voronoi cell [62] of a point is the region of the sphere surface consisting of points which are closer to this given point than to any other ones of the sphere. This cell forms a polygon on this sphere. Each such cell has its area $(A)$ given in the steradian, perimeter $(P)$ given by the length of boundary (one great circle of the boundary curve is also called a "chord"), number of vertices $\left(N_{v}\right)$ given by an integer positive number, and by the inner angles $\left(\alpha_{i} ; i=1, \ldots, N_{v}\right)$. This method is completely non-parametric, and therefore may be sensitive for various point pattern structures in the different subclasses of GRBs.

The points on a sphere may be distributed completely randomly or non-randomly; the non-random distribution may have different characters (clustering, filaments, etc.; for the survey of these nonrandom behaviors, see, e.g., [19]).

The VT method is able both to detect nonrandomness and also to describe its form (see [16, $17,20,34,35,59,62,63,73,74,76]$ for the astronomical context)

\subsection{Minimal spanning tree (MST)}

Unlike VT, this method considers the distances (edges) among the points (vertices). Clearly, there are $N(N-1) / 2$ distances among $N$ points. A spanning tree is a system of lines connecting all the points without any loops. The minimal spanning tree (MST) is a system of connecting lines, where the sum of the lengths is minimal among all the possible connections between the points $[40,58]$. In this paper, the spherical version of MST is used following the original paper by Prim [58].

The $N-1$ separate connecting lines (edges) together define the minimal spanning tree. The statistics of the lengths and the $\alpha_{M S T}$ angles between the edges at the vertices can be used for testing the randomness of the point pattern. The MST is widely used in cosmology for studying the statistical properties of galaxy samples $[1,6-8,21,41]$.

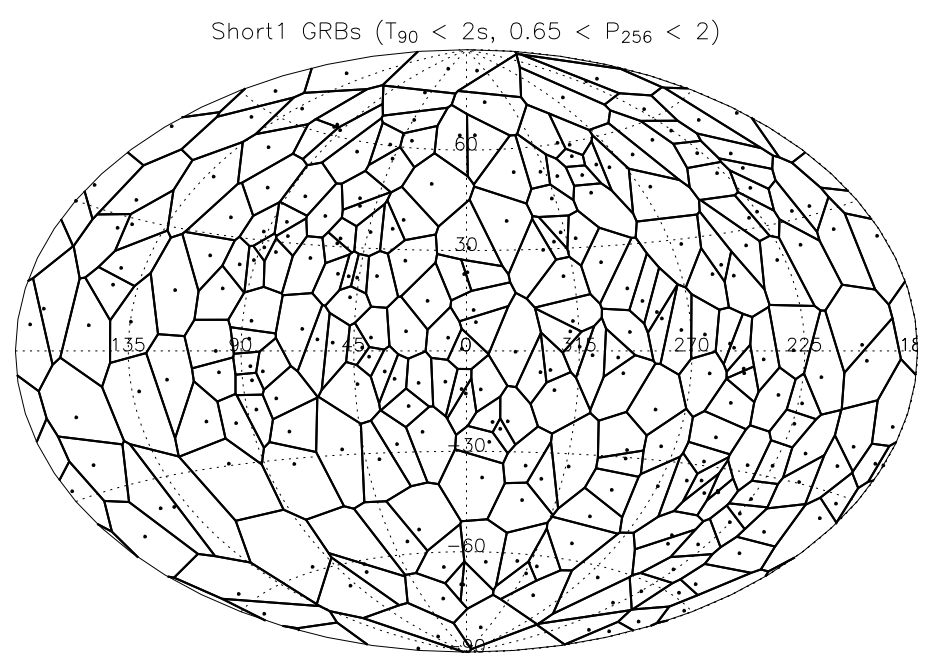

Fig. 1: Application of Voronoi tesselation to short GRBs (Short1 sample) in the $0.65<P_{256}<2.00$ peak flux range in Galactic coordinates. The peak-flux is given in dimension photon $/\left(\mathrm{cm}^{2} \mathrm{~s}\right)$ 


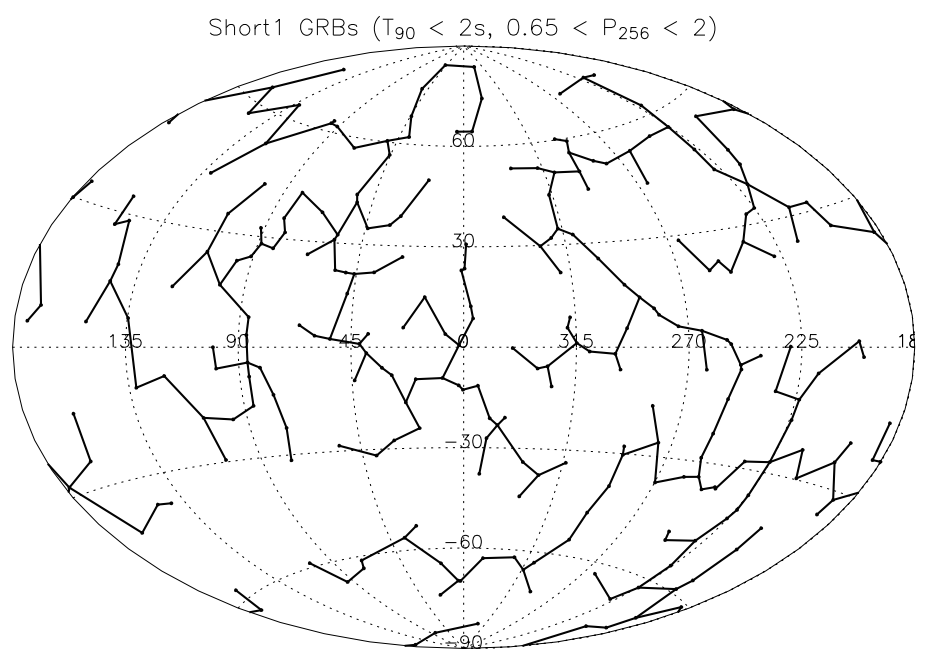

Fig. 2: MST for the sample in Figure 1

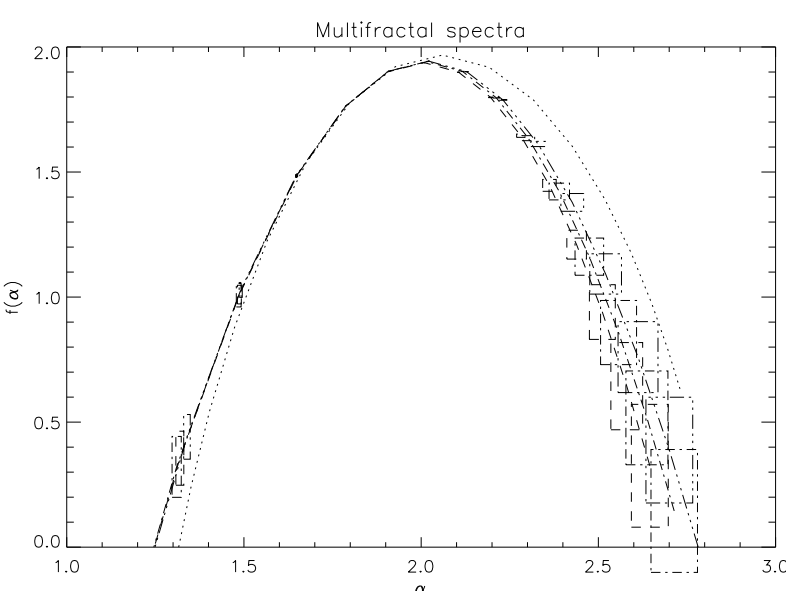

Fig. 3: MFR spectra of simulated (dot-dashed), Long1 (dashed), Short1 (dotted) and Short2 (three-dot-dashed) samples. Boxes represent the error of the spectrum points derived from Monte Carlo simulations. Note the shift of the maximum of the spectrum of the Short1 sample towards higher values in comparison to $\alpha=2$, corresponding to the completely random $2 \mathrm{D}$ Euclidean case

\subsection{Multifractal spectrum}

Let $P(\varepsilon)$ denote the probability for finding a point in an area of $\varepsilon$ radius. If $P(\varepsilon)$ scales as $\varepsilon^{\alpha}$ (i.e. $\left.P(\varepsilon) \propto \varepsilon^{\alpha}\right)$, then $\alpha$ is called the local fractal dimension (e.g. $\alpha=2$ for a completely random process on the plane). In the case of a monofractal $\alpha$ is independent from the position. A multifractal (MFR) on a point process can be defined as unification of the subsets of different (fractal) dimensions [52]. One usually denotes with $f(\alpha)$ the fractal dimension of the subset of points at which the local fractal dimension is in the interval of $\alpha, \alpha+d \alpha$. The contribution of these subsets to the whole pattern is not necessarily equally weighted, in practice, it depends on the relative abundances of subsets. The $f(\alpha)$ functional relationship between the fractal dimension of subsets and the corresponding local fractal dimension is called the MFR or Hausdorff spectrum.

In the vicinity of the $i$-th point $(i=1,2, \ldots, N)$ one can measure from the neighbourhood structure a local dimension $\alpha_{i}$ ("Rényi dimension"). This measure approximates the dimension of the embedding subset, giving the possibility to construct the MFR spectrum which characterizes the whole pattern (for more details see [52]). If the maximum of this convex spectrum is equal to the Euclidean dimension of the space, then in the classical sense the pattern is not a fractal, but the spectrum remains sensitive to the non-randomness of the point set.

The concept of a multifractal can be successfully applied in astronomical problems $[2,9,11-13,18,22$, $25,26,36,37,53,54,60,61,65-67]$.

\section{Evaluation of statistical tests}

The three procedures outlined in Section 2 enable us to derive several stochastic quantities well suited for testing the non-randomness of the underlying point patterns.

\subsection{Input data and sample definition}

Until now the most comprehensive all-sky survey of GRBs was done by the BATSE experiment on board the CGRO satellite in the period from 1991-2000. In this period the experiment collected 2704 well justified burst events, and the data is available in the Current BATSE Catalog [44].

Since there is increasing evidence ( $[31]$ and references therein) that the GRB population is actu- 
ally a mixture of astrophysically different phenomena, we divided the GRBs into three groups: short $\left(T_{90}<2 s\right)$, intermediate $\left(2 s \leq T_{90} \leq 10 s\right)$ and long $\left(T_{90}>10 \mathrm{~s}\right)$. To avoid problems with a changing detection threshold, we omitted GRBs having a peak flux $P_{256} \leq 0.65$ photons $\mathrm{cm}^{-2} \mathrm{~s}^{-1}$. This truncation was proposed in [56]. The bursts may emerge at very different distances in the line of sight and it may happen that the stochastic structure of the angular distribution depends on it. Therefore, we also made tests on the bursts with $P_{256}<2$ photons $\mathrm{cm}^{-2} \mathrm{~s}^{-1}$ in the short and long population, separately. Table 1 defines the 5 samples to be studied here. (Due to the small number of intermediate bursts, this subsample was not divided into faint and bright parts).

Table 1: Tested samples of BATSE GRBs

\begin{tabular}{lllc}
\hline Sample & $\begin{array}{l}\text { Duration } \\
{[\mathrm{s}]}\end{array}$ & $\left.\begin{array}{l}\text { Peak flux } \\
{[\text { photons cm }}\end{array}{ }^{-2} \mathrm{~s}^{-1}\right]$ & $\begin{array}{c}\text { Number } \\
\text { of GRBs }\end{array}$ \\
\hline Short1 & $T_{90}<2 \mathrm{~s}$ & $0.65<P_{256}<2$ & 261 \\
Short2 & $T_{90}<2 \mathrm{~s}$ & $0.65<P_{256}$ & 406 \\
Intermediate & $2 \mathrm{~s} \leq T_{90} \leq 10 \mathrm{~s}$ & $0.65<P_{256}$ & 253 \\
Long1 & $T_{90}>2 \mathrm{~s}$ & $0.65<P_{256}<2$ & 676 \\
Long2 & $T_{90}>10 \mathrm{~s}$ & $0.65<P_{256}$ & 966 \\
\hline
\end{tabular}

\subsection{Definition of the test-variables}

The randomness of the point field on the sphere can be tested with respect to various criteria. Since different non-random behaviors are sensitive to different types of criteria of non-randomness, it is not necessary that all possible tests using different measures reject the assumption of randomness. In the following, we define several test-variables which are sensitive to different stochastic properties of the underlying point pattern, as proposed by [72].

Any of the quantities characterizing the Voronoi cell, i.e. area $A$, perimeter $P$, number of vertices $N_{v}$, cell chord length $C$, and inner angles $\alpha_{i}$ can be used as test-variables, and even some combinations of these quantities. We defined the following testvariables:

- Cell area $A$;

- Cell vertex (edge) $N_{v}$;

- Cell chords length $C$;

- Inner angle $\alpha_{i}$;

- Round factor (RF) average $R F_{a v}=\overline{4 \pi A / P}$;

- Round factor (RF) homogeneity $1-\frac{\sigma\left(R F_{a v}\right)}{R F_{a v}}$;

- Shape factor $A / P^{2}$;

- Modal factor $\sigma\left(\alpha_{i}\right) / N_{v}$;

- The so-called "AD factor" defined as $A D=$ $1-(1-\sigma(A) /\langle A\rangle)^{-1}$, where $\sigma(A)$ is the dispersion and $\langle A\rangle$ is the average of $A$.

To characterize the stochastic properties of a point pattern, we use three quantities obtained from MST:

- Variance of the MST edge-length $\sigma\left(L_{M S T}\right)$;
- Mean MST edge-length $L_{M S T}$;

- Mean angle between edges $\alpha_{M S T}$.

As to the multifractals, the only variable used here is the $f(\alpha)$ spectrum, which is a sensitive tool for testing the non-randomness of a point pattern. Throughout defining these variables mean (average) and variance refer to the mean and variance of the respective elements of the Voronoi foam and MST, respectively.

An important problem is to study the sensitivity (discriminant power) of the different parameters to the different kind of regularity inherent in the point pattern. In the case of a fully regular mesh, e.g., $A$ is constant and so $A D=0, \sigma\left(\alpha_{i}\right)=0$ and both increase towards a fully random distribution. In the case of a patchy pattern the distribution of the area of the Voronoi cells and the edge distribution of MST become bimodal, reflecting the average area and the edge length within and between the clusters, in comparison to the fully random case. In a filamentary distribution, the shape of the areas becomes strongly distorted, reflecting in an increase of the modal factor in comparison to the case of patches.

[71] investigated the power of Voronoi tesselation and the minimal spanning tree in discriminating between distributions having big and small clusters, full randomness and hard cores (random distributions, but the mutual distances of the points are constrained by the size of the hard core), respectively. They concluded that the Voronoi roundness factor did not separate small clusters and hardcore distributions, and the roundness factor homogeneity did not distinguish between small clusters and random distributions, nor between random and hardcore distributions. MST has a very good discriminant power even in the case of hardcore distributions with close minimal interpoint distances.

Since the sensitivity of the variables differs on changing the regularity properties of the underlying point patterns one may measure significant differences in one parameter but not in another, even in cases when these are correlated otherwise. This is not a trivial issue. In most cases, one needs extended numerical simulations to study the statistical significance of the different parameters.

\subsection{Estimation of significance}

To obtain the empirical distributions of the testvariables we made 200 simulations for each of the five samples. The number of simulated points was identical to the number for the samples defined in Section 3.1.

We generated the fully random catalogs by Monte Carlo (MC) simulations of fully random GRB celestial positions and taking into account the BATSE sky-exposure function $[23,44]$. 
Table 2: Calculated significance levels for the 13 test-variables and the five samples. A calculated numerical significance greater than $95 \%$ is put in bold face

\begin{tabular}{llrrrrr}
\hline Name & var & short1 & short2 & interm. & long1 & long2 \\
\hline Cell area & $A$ & 36.82 & 29.85 & 94.53 & 79.60 & 82.59 \\
Cell vertex (edge) & $N_{v}$ & 36.82 & 87.06 & 2.99 & 26.87 & 7.96 \\
Cell chords & $C$ & 47.26 & 52.24 & 18.91 & 84.58 & 54.23 \\
Inner angle & $\alpha_{i}$ & $\mathbf{9 6 . 5 2}$ & 21.39 & 87.56 & 37.81 & 63.18 \\
RF average & $4 \pi A / P$ & 65.17 & $\mathbf{9 9 . 9 8}$ & 33.83 & 10.95 & 86.07 \\
RF homogeneity & $1-\frac{\sigma\left(R F_{a v}\right)}{R F_{a v}}$ & 19.90 & 24.38 & 58.71 & 55.72 & 32.84 \\
Shape factor & $A / P^{2}$ & 91.04 & 94.03 & 90.05 & 55.22 & 63.68 \\
Modal factor & $\sigma\left(\alpha_{i}\right) / N_{v}$ & $\mathbf{9 7 . 5 1}$ & 1.99 & 7.46 & 56.22 & 8.96 \\
AD factor & $1-\left(1-\frac{\sigma(A)}{\langle A\rangle}\right)$ & 32.84 & 25.37 & 11.44 & $\mathbf{9 5 . 5 2}$ & 52.74 \\
MST variance & $\sigma\left(L_{M S T}\right)$ & 52.74 & 38.31 & 22.39 & 13.93 & 59.70 \\
MST average & $L_{M S T}$ & $\mathbf{9 7 . 5 1}$ & 7.46 & 89.05 & 56.72 & 8.96 \\
MST angle & $\alpha_{M S T}$ & 85.07 & 14.43 & 36.82 & 73.63 & 60.70 \\
MFR spectra & $f(\alpha)$ & $\mathbf{9 5 . 5 2}$ & $\mathbf{9 6 . 0 2}$ & $\mathbf{9 8 . 0 1}$ & 73.63 & 36.32 \\
\hline Squared Euclidean & distance & $\mathbf{9 9 . 9 0}$ & $\mathbf{9 9 . 9 8}$ & $\mathbf{9 8 . 5 1}$ & 93.03 & 36.81 \\
\hline
\end{tabular}

Assuming that the point patterns obtained from the five samples defined in Table 2 are fully random, we calculated the probabilities for all the 13 testvariables selected in Section 3.2. Based on the simulated distributions, we computed the level of significance for all the 13 test-variables and in all defined samples.

\section{Discussion of the statistical results}

\subsection{Evaluation of the joint significance levels}

We assigned to each MC simulated sample 13 values of the test variables and, consequently, a point in the 13D parameter space. Completing 200 simulations in all of the subsamples we get in this way a $13 \mathrm{D}$ sample representing the joint probability distribution of the 13 test-variables. Using the Euclidean distance of the points from the sample mean we can get a stochastic variable characterizing the deviation of the simulated points from the mean only by chance. An obvious choice would be the squared Euclidean distance.

In case of a Gaussian distribution with unit variances and without correlations, this would result in a $\chi^{2}$ distribution of 13 degrees of freedom. The testvariables in our case are correlated and have different scales. Before computing squared Euclidean dis- tances we transformed the test-variables into noncorrelated ones with unit variances. Due to the strong correlation between some of the test-variables we may assume that the observed quantities can be represented with non-correlated variables of lower number. Factor analysis (FA) is a suitable way to represent the correlated observed variables with noncorrelated variables of lower number.

Since our test-variables are stochastically dependent, following [72] we attempted to represent them by fewer non-correlated hidden variables, assuming that

$$
X_{i}=\sum_{j=1}^{k} a_{i j} f_{j}+s_{i} \quad i=1, \ldots, p \quad k<p .
$$

In the above equation $X_{i}, f_{j}, s_{i}$ mean the testvariables ( $p=13$ in our case), the hidden variables and a noise-term, respectively. Equation (1) represents the basic model of FA. The covariance matrix of the $X_{i}$ variables has $\frac{1}{2} p(p+1)$ free elements. The number of free elements on the right side of Eq. (1) cannot exceed this value [38], yielding for $k$ the following inequality:

$$
k \leq(2 p+1-\sqrt{8 p+1}) / 2
$$

which gives $k \leq 8.377$ in our case.

Factor analysis is a common ingredient of professional statistical software packages (BMDP, SAS, Splus, SPSS $^{1}$, etc). The default solution for identifying

${ }^{1}$ BMDP, SAS, S-plus, SPSS are registered trademarks 
the factor model is to perform principal component analysis (PCA). We kept as many factors as were meaningful with respect to Equation (2). Taking into account the constraint imposed by Equation (2) we retained 8 factors. In this way we projected the joint distribution of the test-variables in the 13D parameter space into an $8 \mathrm{D}$ parameter space defined by the non-correlated $f_{i}$ hidden variables.

The $f_{j}$ hidden variables in Equation (1) are noncorrelated and have zero means and unit standard deviations. Using these variables, we defined the following squared Euclidean distance from the sample mean:

$$
d^{2}=f_{1}^{2}+f_{2}^{2}+\ldots+f_{k}^{2} \quad(k=8 \text { in our case }) .
$$

If the $f_{j}$ variables had strictly Gaussian distributions, Equation (3) would define a $\chi^{2}$ variable of $k$ degrees of freedom.

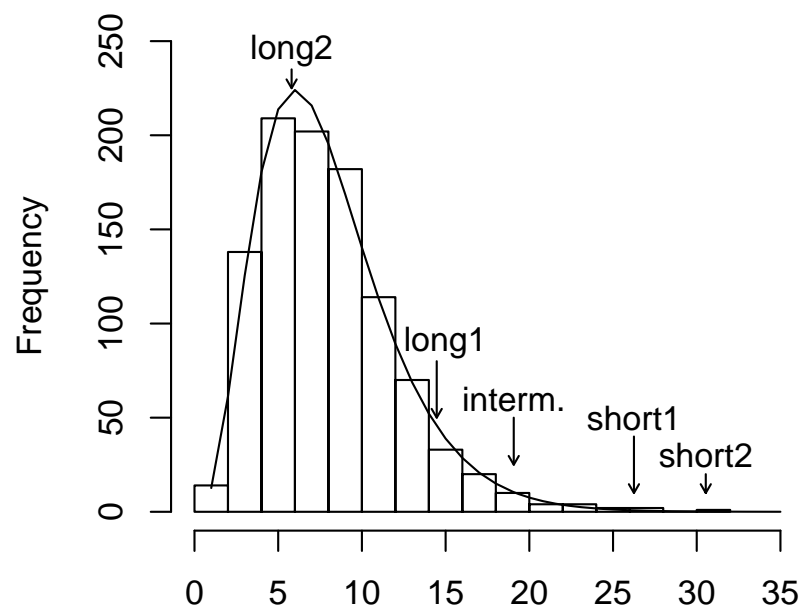

Sq. distance

Fig. 4: Distribution of the squared Euclidean distances of the simulated samples from the stochastic mean of the $f_{i}$ hidden variables (factors) in the $8 \mathrm{D}$ parameter space. There are altogether 1000 simulated points. The full line marks a $\chi^{2}$ distribution of 8 degrees of freedom, normalized to the sample size. The distances of the BATSE samples are also indicated. The departures of samples "short1" and "short2" exceed all those of the simulated points. The probabilities that these deviations are nonrandom equal $99.9 \%$ and $99.98 \%$, respectively

\subsection{Interpretations of the statistical results}

Using the distribution of the squared Euclidean distances, defined by Equation (3), one can get further information on whether a BATSE sample represented by a point in the parameter space of the test-variables deviates only by chance, or whether it differs significantly from the fully random case.
In all categories (short1, short2, intermediate, long1, long2) we made 200, altogether 1000, simulations. We calculated the $d^{2}$ squared distances for all simulations and compared them with those of the BATSE samples in Table 1. Figure 4 shows a histogram of the simulated squared distances along with those of the BATSE samples. A full line represents a $\chi^{2}$ distribution of $k=8$ degrees of freedom. Figure 4 clearly shows that the departures of samples short1 and short2 exceed all those of the simulated points. The probabilities, that these deviations are non-random, equal $99.9 \%$ and $99.98 \%$, respectively.

The full randomness of the angular distribution of the long GRBs, in contrast to the regularity of the short and to some extent to the intermediate ones, points towards the differences in the angular distribution of their progenitors. The recent discovery of the afterglow in some short GRBs indicates that these events are associated with the old stellar population [24] accounted probably for the mergers of compact binaries, in contrast to the long bursts resulting from the collapse of very massive stellar objects in young star forming regions. The differences in progenitors also reflects the differences between the energy released by the short and long GRBs. As [33] showed, the redshift distributions of the different GRB classes are different. The average $z$ of the short bursts is significantly smaller than that of the long ones (the average redshift of intermediate bursts is between them). Consequently, the short and long GRBs experience different sampling volumes. The sampling volume of the short bursts is much smaller and the irregularities in the distribution of the host galaxies plays a more significant role.

Unfortunately, little can be said on the physical nature of the intermediate class. Statistical studies ([31] and the references therein) suggest the existence of this subgroup - at least from the purely statistical point of view. Also a non-random sky distribution occurs here. However, its physical origin is still fully open yet [31].

\section{Summary and conclusions}

We has made additional studies on the degree of randomness in the angular distribution of samples selected from the BATSE Catalog. According to the $T_{90}$ durations and the $P_{256}$ peak fluxes of the GRBs in the Catalog we defined five groups: short1 $\left(T_{90}<2 s \& 0.65<P_{256}<2\right)$, short2 $\left(T_{90}<2 s \&\right.$ $\left.0.65<P_{256}\right)$, intermediate $\left(2 s \leq T_{90} \leq 10 \mathrm{~s} \&\right.$ $\left.0.65<P_{256}\right)$, long1 $\left(T_{90}>2 s \& 0.65<P_{256}<2\right)$ and long2 $\left(T_{90}>10 \mathrm{~s} \& 0.65<P_{256}\right)$.

To characterize the statistical properties of the point patterns given by the samples, we defined 13 test-variables based on Voronoi tesselation (VT), a minimal spanning tree (MST) and a multifrac- 
tal spectra. For all five defined GRB samples we made 200 numerical simulations, assuming fully random angular distribution and taking into account the BATSE exposure function. The numerical simulations enabled us to define the empirical probabilities for testing the null hypothesis, i.e. the assumption that the angular distributions of the BATSE samples are fully random.

Since we performed 13 single tests simultaneously on each subsamples, the significance obtained by calculating it separately for each test cannot be treated as a true indication for deviating from the fully random case. In fact, some of the test-variables are strongly correlated. To concentrate the information on the non-randomness experienced by the test-variables, we assumed that they can be represented as a linear combination of non-correlated hidden factors of lower number. Actually, we estimated $k=8$ as the number of hidden factors. Making use of the hidden factors we computed the distribution of the squared Euclidean distances from the mean of the simulated variables. Comparing the distribution of the squared Euclidean distances of the simulated samples with the BATSE samples we concluded that the short1, short2 groups deviate significantly (99.90\%, 99.98\%) from full randomness, but this is not the case for the long samples. For the intermediate group, squared Euclidean distances also give significant deviation (98.51\%).

\section{Acknowledgement}

This study was supported by OTKA grant No. K077795, by Grant Agency of the Czech Republic grant No. P209/10/0734, and by Research Program MSM0021620860 of the Ministry of Education of the Czech Republic (A.M.).

\section{References}

[1] Adami, C., Mazure, A.: $A \& \&$ AS, 134, 393, 1999.

[2] Aschwanden, M. J., Parnell, C. E.: ApJ, 572, $1048,2002$.

[3] Balázs, L. G., Mészáros, A., Horváth I.: $A \& A$, 339, 1, 1998

[4] Balázs, L. G., Mészáros, A., Horváth, I., Vavrek, R.: A \& A Suppl., 138, 417, 1999.

[5] Balázs, L. G., Bagoly, Z., Horváth, I., Mészáros, A., Mészáros, P.: $A \& \&$ A, 401, 129, 2003.

[6] Barrow, J. D., Bhavsar, S. P., Sonoda, D. H.: MNRAS, 216, 17, 1985.

[7] Bhavsar, S. P., Lauer, D. A.: Examining the big bang and diffuse background radiations, In
Kafatos, M. C. Kondo, Y. (eds.) Proc. IAU Symp. 168, Dordrecht : Kluwer, p. 517, 1996.

[8] Bhavsar, S. P., Splinter, R. J.: $M N R A S, \mathbf{2 8 2}$, $1461,1996$.

[9] Bottorff, M., Ferland, G.: ApJ, 549, 118, 2001.

[10] Briggs, M.: ApJ, 407, 126, 1993.

[11] Casuso, E., Beckman, J.: PASJ, 54, 405, 2002.

[12] Célérier, M. N., Thieberger, R.: $A \& A, \mathbf{3 6 7}$, 449, 2001.

[13] Chappell, D., Scalo, J.: ApJ, 551, 71, 2001.

[14] Cline, D. B., Matthey, C., Otwinowski, S.: Gamma-Ray Bursts: 5th Huntsville Symp., In Kippen, R. M., Mallozzi, R. S., Fishman, G. J. (eds.): AIP Conf. Proc., New York: Melville, p. 97,2000 .

[15] Cline, D. B., Matthey, C., Otwinowski, S.: ApJ, 527, 827, 2000.

[16] Coles, P., Barrow, J. D.: NMRAS, 244, 557, 1990.

[17] Coles, P.: Nature, 349, 288, 1991.

[18] Datta, S.: $A$ \& A, 401, 193, 2003.

[19] Diggle, P. J.: Statistical Analysis of Spatial Point Patterns. London : Academic Press, 1983.

[20] Doroshkevich, A. G., Gottlöber, S., Madsen, S.: A $\&$ A Suppl., 123, 495, 1997.

[21] Doroshkevich, A. G., Turchaninov, V.: In Banday, A. J., Zaroubi, S., Bartelmann, M. (eds.) Proc. 'Mining the Sky' MPA/ESO/MPE Workshop, Springer-Verlag, p. 283, 2001.

[22] Elmegreen, B. G.: ApJ, 564, 773, 2002.

[23] Fishman, G. J., Meegan, C. A., Wilson, R. B., et al.: ApJS, 92, 229, 1994.

[24] Fox, D. B., Frail, D. A., Price, P. A., et al.: $N a-$ ture, 437, 845, 2005.

[25] Gaite, J., Manrubia, S. C.: $M N R A S$, 335, 977, 2002.

[26] Giraud, E.: ApJ, 544, L41, 2000.

[27] Hakkila, J., Haglin, D. J., Pendleton, G. N., et al.: $A p J, \mathbf{5 3 8}, 165,2000$.

[28] Hakkila, J., Giblin, T. W., Roiger, R. J., et al.: ApJ, 582, 320, 2003.

[29] Horváth, I.: ApJ, 508, 757, 1998.

[30] Horváth, I.: $A \&$ \& , 392, 791, 2002. 
[31] Horváth, I., Balázs, L. G., Bagoly, Z., Ryde, F., Mészáros, A.: $A \&$ \& A, 447, 23, 2006.

[32] Horváth, I., Balázs, L. G., Bagoly, Z., Veres, P.: $A \& 6$ A, 489L, 1, 2008.

[33] Horváth, I., Bagoly, Z., Balázs, L. G., de Ugarte Postigo, A., Veres, P., Mészáros, A.: ApJ, 713, 552,2010 .

[34] Icke, V., van de Weygaert, R.: QJRAS, 32, 85, 1991.

[35] Ikeuchi, S., Turner, E. L.: MNRAS, 250, 519, 1991.

[36] Irwin, J. A., Widrow, L. M., English, J.: ApJ, 529, 77, 2000.

[37] Kawaguchi, T., Mineshige, S., Machida, M., Matsumoto, R., Shibata, K.: PASJ, 52, L1, 2000 .

[38] Kendall, M. G., Stuart, A.: The Advanced Theory of Statistics. London - High Wycombe : Charles Griffin \& Co. Ltd., 1973.

[39] Kouveliotou, C., et al.: ApJ, 413, L101, 1993.

[40] Kruskal, J. B.: Proc. Am. Math. Soc., 7, 48, 1956.

[41] Krzewina, L. G., Saslaw, W. C.: $M N R A S, \mathbf{2 7 8}$, 869, 1996.

[42] Magliocchetti, M., Ghirlanda, G., Celotti, A.: MNRAS, 343, 255, 2003.

[43] Litvin, V. F., Matveev, S. A., Mamedov, S. V., Orlov, V. V.: Pis'ma v Astron. Zhurnal, 27, 489, 2001.

[44] Meegan, C. A., et al.: BATSE Gamma-Ray Bursts Catalog. 2000.

http://gammaray.msfc.nasa.gov/batse/grb/ catalog

[45] Mészáros, A., Bagoly, Z., Vavrek, R.: $A \& A$, 354, 1, 2000.

[46] Mészáros, A., Bagoly, Z., Horváth, I., Balázs, L. G., Vavrek, R.: ApJ, 539, 98, 2000.

[47] Mészáros, A., Balázs, L. G., Bagoly, Z., Veres, P.: GAMMA-RAY BURSTS Sixth Huntsville Symposium, 20-23 October 2008, C. Meegan, N. Gehrels, C. Kouveliotou (eds) AIP Conference Proceedings, 1 133, 483, 2009.

[48] Mészáros, A., Balázs, L. G., Bagoly, Z., Veres, P.: Baltic Astronomy, 18, 293, 2009.

[49] Mészáros, P.: Rep. Prog. Phys., 69, 2 259, 2006.
[50] Mukherjee, S., et al.: ApJ, 508, 314, 1998.

[51] Murtagh, F., Heck, A.: Multivariate data analysis, Astrophysics and Space Science Library. Dordrecht : Reidel, 1987.

[52] Paladin, G., Vulpiani, A.: Physics Reports, 156, 1, 1987.

[53] Pan, J., Coles, P.: MNRAS, 318, 151, 2000.

[54] Pan, J., Coles, P.: MNRAS, 330, 719, 2002.

[55] Pásztor, L., Tóth, L. V.: In Shaw, R. A., Payne, H. E., Hayes, J. J. E. (eds) ADASS IV, ASP Conf. Ser. Vol. 77, p. 319, 1995.

[56] Pendleton, C. N., Paciesas, W. S., Briggs, M. S., et al.: ApJ, 489, 175, 1997.

[57] Press, W. H., Flannery, B. P., Teukolsky, S. A., Vetterling, W. T.: Numerical Recipes. Cambridge : Cambridge University Press, 1992.

[58] Prim, R. C.: Bell Syst. Techn. Journ., 36, 1389 , 1957.

[59] Ramella, M., Boschin, W., Fadda, D., Nonino, M.: $A \& A, \mathbf{3 6 8}, 776,2001$.

[60] Selman, F. J., Melnick, J.: ApJ, 534, 703, 2000.

[61] Semelin, B., Combes, F.: $A \&$ \& , 387, 98, 2002.

[62] Stoyan, D., Stoyan, H.: Fractals, Random Shapes and Point Fields. New York: Wiley J. \& Sons, 1994.

[63] Subba Rao, M. U., Szalay, A. S.: ApJ, 391, 483, 1992.

[64] Tanvir, N. R., Chapman, R., Levan, A. J., Priddey, R. S.: Nature, 438, 991, 2005.

[65] Tatekawa, T., Maeda, K.: ApJ, 547, 531, 2001.

[66] Tikhonov, A. V.: Astrophysics, 45, 79, 2002.

[67] Vavrek, R., Balázs, L. G., Epchtein, N.: In Montmerle, T., André, P. (eds) 'From Darkness to Light' ASP Conf. Ser., Vol. 243, p. 149, 2001.

[68] Vavrek, R., Balázs, L. G., Mészáros, A., Horváth, I., Bagoly, Z.: MNRAS, 391, 1741 , 2008.

[69] Veres, P., Bagoly, Z., Horváth, I., Mészáros, A., Balázs, L. G.: ApJ, 725, 1955, 2010.

[70] Voronoi, G.: J. Reine Angew. Math., 134, 198, 1908.

[71] Wallet, F., Dussert, C.: J. theor. Biol., 187, 437, 1997. 
[72] Wallet, F., Dussert, C.: Europhys. Let., 42, 493, 1998.

[73] van de Weygaert, R.: 1994, A \& A, 283, 361.

[74] Yahagi, H., Mori, M., Yoshii, Y.: ApJS, 124, 1, 1999.

[75] Zhang, B., Mészáros, P.: IJMPA, 19, 2385, 2004.

[76] Zaninetti, L.: $A \& A S, \mathbf{1 0 9}, 71,1995$.

L. G. Balázs

Konkoly Observatory

P. O. Box 67

H-1525 Budapest, Hungary

A. Mészáros

Charles University

Faculty of Mathematics and Physics

Astronomical Institute

V Holešovičkách 2, 18000 Prague 8, Czech Republic
I. Horváth

Department of Physics

Bolyai Military University

P. O. Box 15

H-1581 Budapest, Hungary

Z. Bagoly

P. Veres

Department of Physics

Bolyai Military University

P. O. Box 15

H-1581 Budapest, Hungary

Laboratory for Information Technology

Eötvös University

Pázmány Péter sétány $1 / \mathrm{A}$

H-1518 Budapest, Hungary

G. Tusnády

Rényi Alfréd Mathematical Institute

Budapest, Hungary 